\title{
Management of bronchopleural fistula using a modified single lumen tube
}

\author{
Seung Yeon Shin ${ }^{1}$, Hannah Lee ${ }^{1}$, Wooil Kwon ${ }^{2}$, and Ho-Geol Ryu ${ }^{1}$ \\ Departments of ${ }^{1}$ Anesthesiology and Pain Medicine, ${ }^{2}$ Surgery, Seoul National University Hospital, Seoul, Korea
}

Bronchopleural fistula (BPF) is defined as a pathologic connection between the tracheobronchial tree and the pleural space with persistent air leak for more than 24 hours. It is associated with poor prognosis after pneumonectomy [1], which mainly contributes to the occurrence among elective lung resections despite the outstanding advances in surgical techniques. Mechanical ventilation is always problematic for balancing the actual ventilation and the leakage in patients who present a BPF after pulmonary resections. High airway pressure to achieve sufficient minute ventilation may aggravate air leakage and interfere the healing process [2]. We describe a case in which a modified single-lumen endobronchial tube was used in a patient who developed BPF after right lower lung lobectomy in order to improve ventilation and allow adequate suctioning.

A 71-year-old man $(159 \mathrm{~cm}, 48.4 \mathrm{~kg})$ underwent a videoassisted right lower lung lobectomy with mediastinal lymph node dissection for surgical resection of non-small-cell lung cancer. The patient had a previous history of hypertension, diabetes mellitus, chronic renal failure and hepatitis B virus-related cryptogenic liver cirrhosis.

His postoperative course was complicated by persistent air leak through the chest tube. After pleurodesis failed to seal the air leak, serial radiographs had been worsened despite the trial of both antibacterial agents and percutaneous catheter drainage (PCD). A large air leak continued during both inspiration and expiration, combined with dyspnea and producing yellowish sputum. Bronchoscopy and computed tomography (CT) scan showed a definite BPF on the right lower lobar bronchus. Due to recurrent desaturation and failed interventions (PCD repositioning), open window thoracostomy (Eloesser operation) was performed under one-lung ventilation.

On POD 1 after the Eloesser operation, he could not maintain the extubated state and developed aggravating dyspnea and worsening hypoxemia. A 35 French (Fr) left sided double-lumen endotracheal tube was placed and its position was confirmed by bronchoscopy. The left lung was ventilated with nitrous oxide (NO) mixed with air using the synchronized intermittent mandatory ventilation mode with a tidal volume of $270 \mathrm{ml}$. Continuous oxygen flow at $8 \mathrm{~L} / \mathrm{min}$ was applied to the right lung. The patient required frequent tube suctioning. Intravenous midazolam and cisatracurium were infused continuously. Arterial blood gases analysis showed slight improvement afterwards, but worsened over the following 3 days.

On POD 4, the patient showed progressive hypoxemia $\left(\mathrm{SpO}_{2}\right.$ $91 \%)$. Suctioning or manual ventilation with self-inflating resuscitation bag was ineffective. We assessed that the double lumen tube was too narrow to remove the discharges because bronchoscopy showed a thick purulent plug blocking the whole lumen. The tube was changed to a $7.5 \mathrm{~mm}$ single-lumen tube. A thin and long catheter was placed for oxygen supply (5 L/ min) and frequent suctioning (Fig. 1A). The next day, massive air leaks were evident, severely impairing effective ventilation: delivered tidal volumes were $200 \mathrm{ml}$. Bronchoscopy showed that the stump site fistula was exposed and a purulent discharge was flowing over from the right to the left main bronchus. Lung separation was definitely needed, but double lumen tube was not suitable due to productive discharges. Advancing the singlelumen tube would provide both successful lung separation and deep site accessibility (Fig. 1B). Commercial ready-made tubes did not satisfy the purpose; thus, we made a specially devised

Corresponding author: Ho-Geol Ryu, M.D., Department of Anesthesiology and Pain Medicine, Seoul National University Hospital, 101, Daehangno, Jongno-gu, Seoul 110-744, Korea. Tel: 82-2-2072-2469, Fax: 82-2-747-5639, E-mail: hogeol@gmail.com

(c) This is an open-access article distributed under the terms of the Creative Commons Attribution Non-Commercial License (http:// creativecommons.org/licenses/by-nc/3.0/), which permits unrestricted non-commercial use, distribution, and reproduction in any medium, provided the original work is properly cited. 

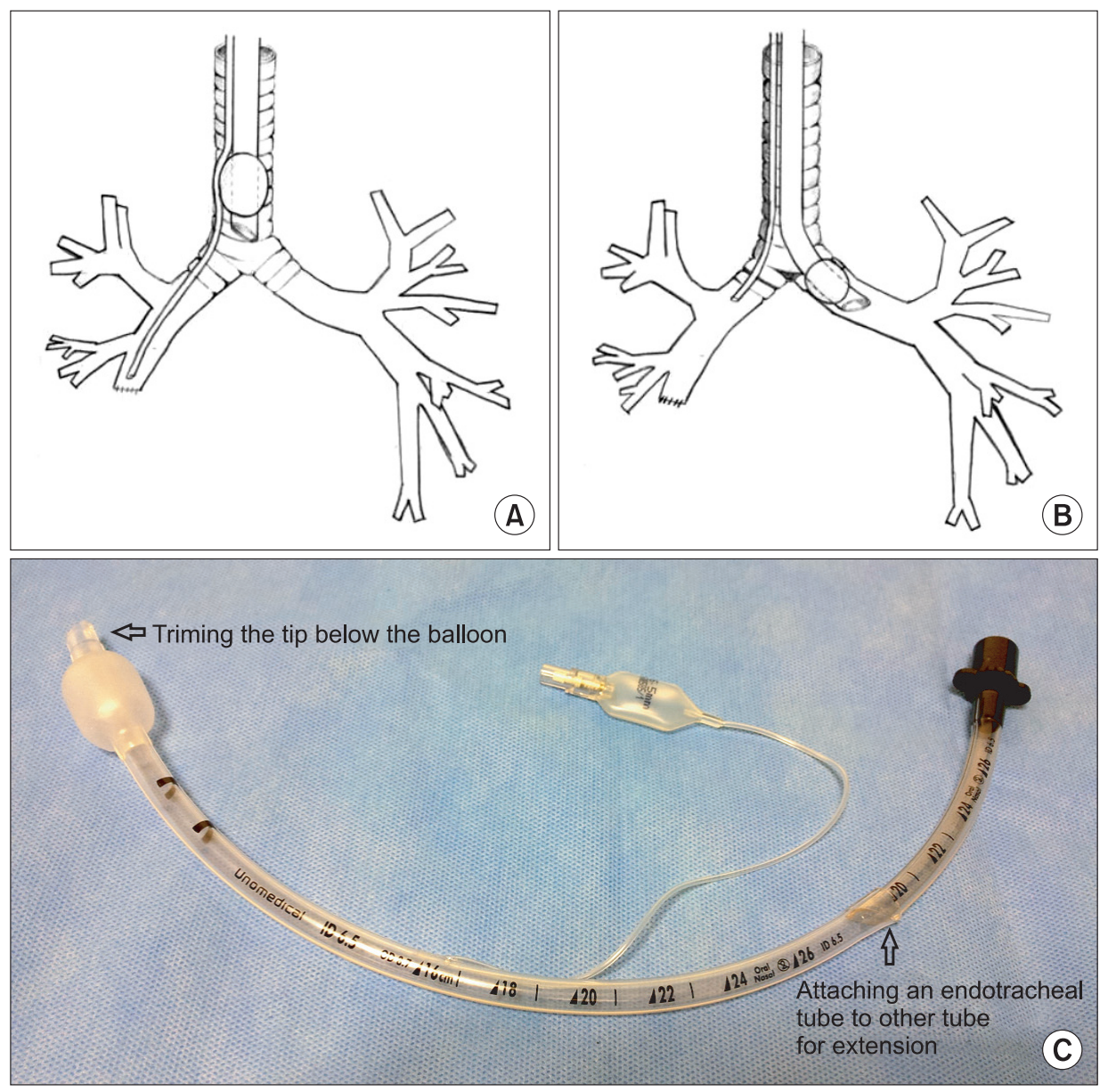

Fig. 1. (A) A single-lumen endobronchial tube and a suction catheter were placed; (B) then the tube was exchanged with a modified endobronchial tube. (C) The features were specified. endotracheal tube, which was made by attaching an endotracheal tube to the other tube for extension and trimming the tip below the balloon to be placed above the left upper bronchus (Fig. 1C). The left lung was isolated successfully, while the right bronchial catheter remained. Oxygen delivery rate through the catheter was increased to $10 \mathrm{~L} / \mathrm{min}$. Almost a complete disappearance of the air-leak and a progressive re-expansion of the left lung were obtained, with the entire tidal volume returned with expiration. Mechanical ventilation was maintained for another 8 days. Despite meticulous airway management, the patient died from aggravated aspiration pneumonia.

The conventional management of BPF is to close it surgically, but this is not always possible and may not be the best option. Patients who develop BPF often have a history of repeated surgery and poor functional status and subsequently, often lack the reserve to withstand additional surgical procedures. Sealants via bronchoscopy have been developed to lighten the physical burden [3]. For fistulas smaller than $8 \mathrm{~mm}$ in diameter, bronchoscopic treatments have been reported to be successful. Santini and colleagues reported a case series that described the successful use of a specially preformed double bronchial balloon double lumen tube (sher-i-bronchi ${ }^{\odot}$, Sheridan catheter corp., Argyle, USA) by diverting around the right upper bronchial lobectomy stump.

The size and location of the BPF as well as the general condition of the patient should be considered carefully when establishing the treatment plan [4]. Our patient required surgical repair because the stump fistula was too large. In addition, alternative methods were not sufficient to provide the required ventilation. Double-lumen tubes, such as the ones Santini et al. used, or Endobronchial blockers were not applicable because the patency of the lumen was not maintained, owing to the thick purulent sputum. Alternative approaches using fiberoptic bronchoscopy were not indicated due to the size of the BPF. Our experience demonstrates the effectiveness of a modified endobronchial tube designed to satisfy both differential ventilation and suction.

In conclusion, the successful management of a bronchopleural fistula depends on formulating a tailored treatment strategy. Although the final result was disappointing, we describe a case of BPF in which the endotracheal tube was modified to meet the required needs. 


\section{References}

1. Sirbu H, Busch T, Aleksic I, Schreiner W, Oster O, Dalichau H. Bronchopleural fistula in the surgery of non-small cell lung cancer: incidence, risk factors, and management. Ann Thorac Cardiovasc Surg 2001; 7: 330-6.

2. Luks AM, Pierson DJ. Barotrauma and bronchopleural fistula. In : Principles and Practice of Mechanical Ventilation. 23rd ed. Edited by Tobin MJ: New York, McGraw-Hill, 2012, pp 943-61.

3. Lois M, Noppen M. Bronchopleural fistulas: an overview of the problem with special focus on endoscopic management. Chest 2005; 128: 3955-65.

4. Shekar K, Foot C, Fraser J, Ziegenfuss M, Hopkins P, Windsor M. Bronchopleural fistula: an update for intensivists. J Crit Care 2010; 25 : 47-55. 